\title{
Sobre modelagem matemática e formalismos estatísticos de sistemas complexos
}

\author{
Mathematical modeling and statistical formalisms of complex systems \\ Clóves G. Rodrigues ${ }^{*}$, Fabio S. Vannucchi², Roberto Luzzi ${ }^{3}$ \\ ${ }^{1}$ Pontifícia Universidade Católica de Goiás, Escola de Ciências Exatas e da Computação, Goiânia, GO, Brasil \\ ${ }^{2}$ Universidade Estadual Paulista, Instituto de Biociências do Campus do Litoral Paulista, São Paulo, SP, Brasil \\ ${ }^{3}$ Universidade Estadual de Campinas, Campinas, SP, Brasil
}

Recebido em 19 de Abril de 2019. Revisado em 31 de Maio de 2019. Aceito em 13 de Julho de 2019

\begin{abstract}
Os Sistemas Dinâmicos Complexos são sistemas com propriedades emergentes inesperadas resultantes de processos sinérgicos de seus componentes. Estes sistemas aparecem na física e na química e desempenham um papel fundamental em sistemas biológicos. Tais sistemas requerem um tratamento teórico em termos de "Modelagem Matemática", com os formalismos estatísticos sendo de grande relevância. Apresentamos aqui um artigo explanatório descrevendo e discutindo o tema. Apresentamos uma revisão do assunto com particular atenção à Teoria da Informação em uma abordagem de Shannon-Jaynes no espírito da proposta de inferência científica de Jeffreys. Enfatiza-se a difícil questão da presença das "hidden constraints" e a introdução de estatísticas não convencionais que surgem no âmbito da "Teoria da Informação".
\end{abstract}

Palavras-chave: formalismos estatísticos, modelos matemáticos, estatísticas não-convencionais.

The "Complex Dynamical Systems" are systems with unexpected emerging properties which are the result of synergetic processes of their components. These systems appear in condensed matter physics and chemistry, as well as, playing a fundamental role, in biological systems. They require a theoretical treatment in terms of "Mathematical Modelling", with statistical formalisms being of large relevance. We present here a feature-like article describing and discussing the question. Particular attention has been done on the basis of Information Theory in a Shannon-Jaynes approach in the spirit of Jeffreys' scientific inference proposal. It is emphasized the difficult question of presence of "hidden constraints", and the introduction of nonstandard statistics arising in the realm of "Information Theory".

Keywords: statistical approach, nonstandard statistics, information theory.

\section{Introdução}

Há mais de trinta anos E. W. Montroll e M. F. Shlesinger escreveram que: “... no mundo da investigação de fenômenos complexos que exigem modelagem estatística e interpretação, vários estilos concorrentes surgiram, cada um com seus próprios defensores"[1]. De fato, no estudo de certos sistemas físico-químicos podemos enfrentar dificuldades no tratamento de algumas situações como, por exemplo, as envolvendo estruturas tipo fractais, correlações (espaciais e temporais) com algum tipo de escala, movimentos turbulentos ou caóticos, dimensões reduzidas (escala nanométrica). Assim, é nessas situações que nos deparamos com a existência de "hidden constraints" às quais não temos acesso.

O interesse no estudo destes tipos de sistemas físicos complexos tem sido aprimorado recentemente como consequência do fato que eles estão presentes na física de dispositivos eletrônicos e optoeletrônicos utilizados nas avançadas tecnologias atuais, e também em áreas

*Endereço de correspondência: cloves@pucgoias.edu.br tecnológicas/industriais envolvendo o uso de sistemas desordenados, soluções e materiais poliméricos, condutividade iônica em vidros, o caso de micro-baterias e outros. Também é de grande relevância a questão da emergente, e em grande expansão, Biologia de Sistemas [2], envolvendo complexidade, auto-organização e teoria da informação.

A Teoria dos Sistemas Dinâmicos é considerada pioneira por L. von Bertalanffy, que escreveu: “... se alguém analisasse as noções atuais e as palavras de ordem da moda, encontraria a palavra 'sistemas' no topo da lista. Este conceito permeou todos os campos da ciência e penetrou no pensamento popular, jargão e mídia de massas". Esta observação está na Introdução do livro de Bertalanffy de 1967 [3], o qual é uma compilação de seus escritos, sendo as primeiras contribuições datadas de meados da década de 1930.

A Modelagem Matemática é hoje parte integrante das pesquisas atuais em biologia, particularmente em biologia celular em nível molecular e biologia de sistemas, tentando formular e resolver muitos dos problemas im- 
portantes e desconcertantes da biologia, como aqueles que lidam com regulação e controle no sentido mais amplo [4]. Em particular, podemos mencionar a modelagem celular, uma tarefa desafiadora da biologia de sistemas e da biologia matemática; simulação de organismos multicelulares; predição de dobragem de proteínas da sua estrutura tridimensional a partir da sua sequência de aminoácidos; modelos do cérebro até o nível molecular; modelos do sistema imunológico; o fígado virtual, um projeto para produzir um modelo matemático dinâmico representando o fígado humano; modelos ecológicos, isto é, representação matemática de ecossistemas; modelos em ecotoxicologia, ou seja, simulação e previsão de efeitos produzidos por compostos tóxicos no meio ambiente; modelagem de doenças infecciosas para prever o provável resultado de uma epidemia; etc.

Outro tópico importante envolvendo a modelagem matemática é o da geração espontânea de padrões espaciais, originada por A. Turing [5]. Alan Turing estabeleceu a base para computadores modernos com sua análise dos requisitos lógicos para Algoritmos em sua máquina de Turing, sendo também o homem que formulou a ideia fundamental sobre o surgimento de padrões em organismos $[6]$.

A seguir, enfatizamos os aspectos das abordagens estatísticas, e encerramos essa Introdução chamando a atenção para a descrição de modelagem de sistemas biológicos no seguinte link:

http://en.wikipedia.org/wiki/modelling/_/_biological/ __systems

onde são dadas extensas referências de pesquisas publicadas sobre este tema.

\section{As abordagens estatísticas}

Como afirmado no início da Introdução na seção anterior, a estatística desempenha um papel fundamental na área de Modelagem Matemática de Sistemas, particularmente, na física da matéria condensada [7] e na biologia [8].

A complexidade está presente em muitos casos (autoorganização é a regra em sistemas biológicos) envolvendo em várias ocasiões a dificuldade de ter que enfrentar a presença de "hidden constraints", isto é, não ter acesso à informação que é bastante relevante para a descrição apropriada das propriedades do sistema. A questão envolve o fato que o pesquisador enfrenta dificuldades em satisfazer os critérios de eficiência e suficiência de R. A. Fisher [9] na abordagem convencional da bem estabelecida, física e logicamente sólida estatística de Boltzmann-Gibbs. O critério de eficiência é satisfeito por aquelas estatísticas que, obtidas de grandes amostras, tendem a uma distribuição normal com o menor desvio padrão possível. Uma estatística satisfaz o critério de suficiência quando nenhuma outra estatística a qual pode ser calculada a partir da mesma amostra fornece alguma informação adicional como o valor do parâmetro a ser estimado que, para efeitos de mecânica estatística, deve ser considerado como a existência de uma descrição incompleta da situação física em mãos [10]. Normalmente, na Mecânica Estatística, a questão consiste na necessidade de confrontar com algumas deficiências como, por exemplo, em como incluir corretamente a presença de grandes flutuações (e, eventualmente, variações de ordem mais alta) e considerar as características relevantes e apropriadas do sistema implicando na falta de eficiência e suficiência, respectivamente. Como consequência, na tentativa de amenizar essas dificuldades, e assim permitir melhorar as previsões, pode-se recorrer a abordagens estatísticas diferentes das consistentes e, digamos, canônicas, de Boltzmann-Gibbs.

Entre as abordagens existentes, podemos mencionar:

1. A utilizada por P. T. Landsberg mostrando que as propriedades funcionais das entropias (informacionais) dão origem, de fato, a diferentes tipos de termodinâmicas não usuais, e levantam a questão de como selecionar uma termodinâmica "adequada", isto é, são algumas melhores que outras? [11];

2. A Estatística de Lèvy tem sido utilizada por décadas, introduzindo distribuições não gaussianas modificadas, que tem sido aplicadas a uma variedade de problemas, e recentemente teve um renascimento com sua aplicação ao estudo da dinâmica caótica (ver, por exemplo, Refs. $[1,12,13]$ );

3. A abordagem de W. Ebeling $[14,15]$, que explanou a questão do tratamento estatístico de uma classe de sistemas que são em certo sentido anômalos. Eles contêm aqueles na natureza e na sociedade que são determinados por sua história total. Geralmente os exemplos dados são a evolução do Universo e do nosso planeta, fenômenos nos níveis biológico, ecológico, climático, social, etc. A abordagem consiste em introduzir probabilidades condicionais no contexto do formalismo de Boltzmann-Gibbs na abordagem de Shannon-Jaynes, levando a uma termo-estatística apropriada para descrever processos complexos com memória de longo alcance e incluindo correlações [14-16].

4. A chamada "Super-Estatística" desenvolvida por C. Beck e E. G. D. Cohen para sistemas fora do equilíbrio com dinâmica complexa em estados estacionários com grandes flutuações em escalas de longo tempo [17];

5. A geralmente chamada "Termo-Estatística Não Extensiva", baseada na abordagem Havrda-Charvat [18], que se aplica a vários casos, é bem descrita nos Anais da Conferência da Ref. [19]; ainda neste contexto podemos citar os trabalhos de C. Tsallis, sendo o seu trabalho principal publicado em 1988 [20], com uma vasta bibliografia de artigos relacionados diretamente ao seu trabalho por cientistas de todo o mundo disponível em: http: //tsallis.cat.cbpf.br/biblio.htm

6. A "Abordagem de A. Renyi" [21,22] foi introduzida na literatura científica de forma modificada (como observado na Ref. [23]) por P. Grassberger e 
I. Procaccia $[24,25]$ usando-a como um método valioso para caracterizar sinais caóticos experimentais. P. Jizba e T. Arimitzu apresentaram uma extensa análise dela em um artigo intitulado " $O$ mundo de acordo com Renyi", onde mostram que maximizar a entropia de Shannon em um multi-fractal é equivalente a maximizar diretamente a entropia de Renyi, sem invocar explicitamente a estrutura multi-fractal [26];

7. A abordagem de Sharma-Mittal [27], ou melhor dizendo, uma variação dela (chamada Kappa ou Estatística Deformacional), foi usada por V. M. Vasyliunas em problemas de plasmas astrofísicos [28] e por G. Kaniadakis no caso de sistemas relativísticos [29].

Lembramos que na Mecânica Estatística a distribuição de probabilidade (ou operador estatístico na formulação quântica), geralmente obtida de argumentos heurísticos, também pode ser formulada por uma condição de extremo uma vez que seja feita uma conexão com a Teoria da Informação [30-32]. Basicamente consiste em maximizar, sujeito a certas restrições, um funcional. Essa quantidade, introduzida pela primeira vez na Teoria $d a$ Comunicação de Shannon [33], pode ser referida como "medida da incerteza da informação". Também tem sido chamada de medida estatística e entropia, com o entendimento de que é "entropia teórica de informação". Vale a pena enfatizar - em vista de alguma confusão que recentemente penetrou na literatura científica - que as possíveis diferentes entropias da informação não devem ser interpretadas como a entropia termodinâmica do sistema físico. R. T. Cox notou que o significado de tais entropias não é o mesmo em todos os aspectos como o de qualquer coisa que tenha um nome familiar de uso comum e, portanto, é impossível dar uma simples descrição verbal dele, que é, ao mesmo tempo, uma definição precisa [34]. E. T. Jaynes também comentou que existe uma falha persistente em não distinguir entre a entropia informacional, que é uma propriedade de qualquer distribuição de probabilidade, e a entropia experimental da termodinâmica, que é uma propriedade de um estado termodinâmico. Muitos trabalhos de pesquisa são fatalmente falhos pelo erro dos autores em não distinguir entre essas definições inteiramente diferentes e, em consequência, provam resultados sem sentido [35,36].

A entropia teórica da informação de Gibbs-BoltzmannShannon (medida de Kullback-Leibler na Teoria da Informação [37]) é dada por:

$$
S_{\mathrm{GBS}}(t)=-\operatorname{Tr}\{\varrho(t) \ln \varrho(t)\}
$$

onde $\varrho(t)$ é o operador estatístico para o correspondente ensemble de Gibbs. A obtenção do operador estatístico $\varrho(t)$ foi feita usando vários argumentos de consistência (ver, por exemplo, o artigo de revisão de R. Balian e N. L. Balazs da Ref. [38]). Entre eles, podemos destacar aquele baseado em argumentos heurísticos (como é usual nos livros textos para o caso de equilíbrio; e para sistemas de não-equilíbrio ver, por exemplo, Refs. $[39,40])$, e aquele que usa um princípio de abordagem extremal que consiste em maximizar a $S_{\mathrm{GBS}}(t)$ sujeita a restrições (o chamado formalismo "MaxEnt") [30,31].

No que diz respeito à Mecânica Estatística de sistemas de muitos corpos, pode-se notar que ela tem uma longa e bem-sucedida história. A introdução do conceito de probabilidade na física originou-se principalmente do ensaio fundamental de Laplace [41], que incorporou e ampliou algumas ideias seminais anteriores (ver uma revisão histórica na Ref. [42]). Como é sabido, a Mecânica Estatística atingiu o status de uma disciplina bem estabelecida nas mãos de Maxwell, Boltzmann, Gibbs e outros, e passou por algumas etapas relacionadas a mudanças, não em sua estrutura fundamental, mas apenas no substrato fornecido pela mecânica microscópica. Sua aplicação ao caso de sistemas em equilíbrio procedeu-se rapidamente e com excepcional sucesso. A mecânica estatística de equilíbrio forneceu - a partir do nível microscópico - a base para a Termostática, e a possibilidade de construir uma Teoria da Função Resposta. Aplicações a sistemas de não-equilíbrio começaram, principalmente, com o caso do equilíbrio local no regime linear seguindo o trabalho pioneiro de L. Onsager [43] (veja também Ref. [44]).

Para sistemas arbitrariamente fora do equilíbrio e governados por leis cinéticas não-lineares, o desenvolvimento de um formalismo como ensemble progrediu em um ritmo bem mais lento que no caso do equilíbrio, e um tanto cautelosamente, com uma longa lista de cientistas ilustres contribuindo para tal desenvolvimento. Percebese que a Mecânica Estatística ganhou nos anos 50 uma abordagem alternativa sustentada nas bases da Teoria da Informação $[31,39,40,42,45-50]$ e invocou as ideias da Teoria da Informação acompanhadas de ideias de inferência científica [51,52], e um formalismo de princípio extremo (sendo este último o princípio de maximização da incerteza informacional de Jaynes - também referido como entropia informacional - abreviado como "MaxEnt"), compondo de tal ponto de vista uma teoria chamada de Mecânica Estatística Preditiva [31,32,42,45-49]. Deve-se notar que este não é um novo paradigma na Física Estatística, mas um princípio extremo, útil e prático que codifica a obtenção de distribuições de probabilidade, que geralmente são obtidas por abordagens heurísticas ou técnicas de operadores de projeção $[30,52,53]$. É particularmente vantajoso construir ensembles estatísticos de não-equilíbrio que permitam a sistematização dos trabalhos relevantes sobre o assunto que cientistas renomados forneceram ao longo do século passado, conforme descrito na Ref. [30].

Entretanto, foi notado que o "MaxEnt" não é um princípio físico no sentido próprio, e deve ser cuidadosamente distinguido do "princípio de máxima entropia" da termodinâmica. Este último não é uma regra de inferência, mas uma condição de equilíbrio termodinâmico [54]. O "MaxEnt", aparentemente proposto inicialmente por W. 
Elssasser [55], foi sistematizado e analisado em profundidade por E. T. Jaynes [46-48], que o propôs como uma extensão do princípio da razão insuficiente na Lógica, alegando que o "MaxEnt" é um método único de inferência estatística concordando com certos requisitos convincentes de consistência. Este ponto foi criticamente revisado por J. Uffink [54]. Além disso, R. Landauer argumentou que "... o MaxEnt é sólido, mas muitas vezes é terrivelmente difícil entender quais são as restrições" [56]. Mario Bunge afirmou que: "... quando confrontado com um processo aleatório ou aparentemente aleatório, tenta-se construir um modelo probabilístico que possa ser testado contra dados empíricos, sem aleatoriedade, sem probabilidade" [57]. Além disso, como H. Poincaré apontou há muito tempo, falar de probabilidade envolve algum conhecimento; não é um substituto para a ignorância. Bunge acrescenta, não corretamente, no que se refere à mecânica estatística, que: “... não é assim que o Bayesiano ou um ponto de vista pessoal vê o assunto quando confrontado com a ignorância ou incerteza, eles usam probabilidade - ou melhor, sua própria versão. Isso permite que eles atribuam probabilidades anteriores a fatos e proposições de uma maneira arbitrária [de novo, esse não é o caso do Formalismo do Ensemble estatístico de não-equilíbrio baseado no "MaxEnt"] - que é uma maneira de passar a mera intuição, palpite ou pressentimento para hipótese científica [...]; é todo um jogo de crença e não de conhecimento" [57]. Argumentos contra o "MaxEnt" em relação a jogar dados foram apresentados. A isto, deve ser lembrado que a questão que estamos abordando aqui não lida com o jogo, mas com a teoria de muitos corpos, ou seja, lidamos com sistemas com muitos graus de liberdade e, em seguida, é necessário ter em mente a distinção entre interpretações em termos de variáveis microscópicas e macroscópicas.

Citando P. S. Laplace: “... a curva descrita por uma molécula de ar ou de vapor segue uma regra tão certamente quanto as órbitas dos planetas: a única diferença entre as duas é nossa ignorância. A probabilidade está relacionada, em parte a essa ignorância, em parte ao nosso conhecimento" [41]. Além disso, como apontado por J. Bricmont: “... a parte 'devida à nossa ignorância' é simplesmente que usamos o raciocínio probabilístico. Se fôssemos oniscientes, não seria necessário (mas as médias permaneceriam o que são, é claro). A parte 'devida ao nosso conhecimento' é o que faz o raciocínio funcionar [...]. Mas é assim que as coisas são: nosso conhecimento é incompleto e temos que viver com isso. No entanto, o raciocínio probabilístico é extraordinariamente bemsucedido na prática, mas, quando funciona, isso se deve ao nosso conhecimento (parcial). Seria errado atribuir qualquer papel construtivo à nossa ignorância. E também é errado supor que o sistema deve ser de alguma forma indeterminado quando aplicamos o raciocínio probabilístico a ele" [58] (ver também Ref. [59]). Deve-se notar que deduzir o comportamento do estado macroscópico do sistema do conhecimento parcial já estava presente no brilhante trabalho original de J. W. Gibbs.

W. Heisenberg escreveu: "Gibbs foi o primeiro a introduzir um conceito físico que só pode ser aplicado a um objeto quando nosso conhecimento do objeto é incompleto" [60]. Além disso, pode-se considerar que a rejeição de uma abordagem teórica na Física não pode (e não deve) ser feita com base em argumentos verbais gerais, que podem ou não ser sensíveis, mas que precisam ser fortemente fundamentados no método científico. Em outras palavras, os méritos, ou melhor, os deméritos, de uma teoria residem no estabelecimento de seu domínio de validade quando testados em relação aos resultados experimentais que a referida teoria prevê [61,62]. Este ponto foi enfatizado também por S. W. Hawking [63].

No caso particular da abordagem do princípio de maximização no estilo de E. T. Jaynes (como um caminho alternativo [40] para, por exemplo, o heurístico [64] para construir o Formalismo do Ensemble Estatístico de NãoEquilíbrio) L. Sklar resumiu que: “... em primeiro lugar Jaynes sugeriu que a mecânica estatística de equilíbrio pode ser vista como um caso especial do programa geral de raciocínio indutivo sistemático e que, desse ponto de vista, as distribuições de probabilidade introduzidas na mecânica estatística têm suas bases não tanto em uma investigação empírica de ocorrências no mundo, mas, em vez disso, em um procedimento geral para determinar as probabilidades subjetivas a priori apropriadas de uma maneira sistemática" [65].

Análises adicionais estão presentes no trabalho de B. C. van Fraassen [66-68], onde são discutidas as possibilidades de regras alternativas de construção de expressões de entropia generalizadas (teoria da informação) contendo um parâmetro contínuo livre (as chamadas Entropia de Renyi). J. Uffink notou que parece que mais pesquisas seriam necessárias para avaliar seu desempenho em casos concretos e em geral. Além disso, em um artigo intitulado "Entropies Galore!" [11], P. T. Landsberg chamou a atenção para o fato de que muitas dessas entropias teóricas de informação podem ser propostas (veja Ref. [69]).

A abordagem informacional tem sido bastante bem sucedida em sua aplicação aos casos de condições de equilíbrio e quase equilíbrio $[39,40,45,46]$, e nas últimas décadas tem sido, e está sendo, aplicada também à construção de uma teoria de ensemble generalizado para sistemas arbitrariamente longe do equilíbrio [30,52,53,70-73]. O Formalismo do Ensemble Estatístico de Não Equilíbrio "NESEF" fornece fundamentos estatísticos-mecânicos à termodinâmica irreversível (na forma da Termodinâmica Estatística Informativa, abreviada por "IST" [74-77]), a uma teoria cinética quântica não-linear [30,52,53,78-81] e a uma teoria da função resposta $[30,82]$ de um grande escopo para lidar com sistemas de muitos corpos arbitrariamente distantes do equilíbrio. O "NESEF" tem sido aplicado com sucesso ao estudo de várias situações de não-equilíbrio na física de semicondutores [7] e polímeros 
[83], bem como em estudos de comportamento complexo de sistemas bosônicos em biopolímeros [8] e sistemas de fônons $[84,85]$. Também pode ser notado que a teoria cinética não-linear baseada no "NESEF" fornece, em casos limites particulares, generalizações de longo alcance das equações de L. Boltzmann [86] e H. Mori [87], juntamente com fundamentos estatísticos para a Termodinâmica Irreversível Mesoscópica [88], e uma hidrodinâmica de ordem superior [89-93].

O "NESEF" é construído sobre as bases de argumentos de padrão heurísticos (ver, por exemplo, Ref. [64]), ou dentro do escopo do método do princípio extremo baseado na maximização da entropia de Shannon-Jaynes na abordagem estatística de Boltzmann-Gibbs dada pela Eq. (1), isto é, a média de menos o logaritmo do operador estatístico de não-equilíbrio dependente do tempo (isto é, dependente da evolução irreversível do estado macroscópico do sistema). Novamente enfatizamos que a entropia da teoria da informação é, de fato, a quantidade de incerteza da informação, e tem o papel de um funcional a partir do qual obtêm-se as distribuições de probabilidade para lidar com problemas na Teoria da Comunicação, Física, Matemática Econômica, etc. Há uma e apenas uma situação na qual a entropia informacional de Shannon-Jaynes coincide com a entropia física de Clausius na termodinâmica, a saber, o caso do equilíbrio estrito $[11,94]$.

Já chamamos a atenção para o artigo clássico e fundamental de 1922 de R. A. Fisher, intitulado "On The Mathematical Foundations of Theoretical Statistics" [9], onde são apresentados os critérios básicos que uma estatística deve satisfazer para fornecer resultados valiosos. Reiteramos que nos dias atuais na Mecânica Estatística dois critérios básicos são de grande relevância, a saber, o "critério de eficiência" e o "critério de suficiência", já descritos. Isto é assim devido a restrições particulares que estão presentes, por exemplo, em situações físicas envolvendo sistemas muito pequenos, onde por um lado o número de graus de liberdade que entram na estatística pode ser pequeno, e por outro lado a presença de estruturas, incluindo condições de contorno de caráter tipo fractal que influenciam fortemente as propriedades do sistema. Tais fatos dificultam a introdução de informações suficientes para obter uma distribuição de probabilidade adequada do tipo Boltzmann-Gibbs, e podemos mencionar os exemplos de nanotecnologia, nanobiofísica, pontos quânticos e heteroestruturas nanométricas em dispositivos semicondutores, transistores de uma molécula, eletrodos fractais em microbaterias, etc. Outro caso em que o critério de suficiência é difícil de satisfazer é o de grandes sistemas de fluidos cujo movimento hidrodinâmico está além do domínio de validade da abordagem padrão clássica. É então necessário o uso de uma hidrodinâmica de ordem superior não linear, eventualmente incluindo correlações e outras variações (um exemplo típico é o caso do movimento turbulento). Isto tem uma certa analogia no tratamento de biossistemas.

\section{Comentários finais}

Os chamados Sistemas Dinâmicos Complexos (uma abreviação para sistemas que exibem comportamento complexo) são sistemas com propriedades emergentes inesperadas que são o resultado de processos sinérgicos de seus componentes, cujo tratamento depende em grande parte da modelagem matemática e das abordagens estatísticas associadas.

Apresentamos uma revisão do tema com ênfase dada na abordagem estatística associada. Particular atenção tem sido feita com base na Teoria da Informação em uma abordagem de Shannon-Jaynes no espírito da proposta de inferência científica de Jeffreys.

Alguma atenção também foi dada às dificuldades trazidas pela inconveniência da presença de "hidden constraints". Alguns comentários estão incluídos na questão do uso de estatísticas não convencionais, particularmente a de Renyi.

Destacamos neste artigo que os fundamentos da Mecânica Estatística na Teoria da Informação têm sido bem sucedidos em suas aplicações às situações de equilíbrio e condições de equilíbrio próximo, e estão sendo usados na construção de uma teoria de ensemble generalizada para sistemas arbitrariamente distantes do equilíbrio envolvendo processos de relaxamento ultra-rápidos no tempo (escalas pico e femto-segundo) e em regiões ultrapequenas (escalas nanométricas).

\section{Referências}

[1] E.W. Montroll e M.F. Shlesinger, Journal of Statistical Physics 32, 209 (1983).

[2] L. Chong e L.B. Ray, Science 295, 1589 (2002).

[3] L. Bertalanffy, General System Theory Foundations, Development, Applications (New York, G. Braziller Inc., 1968).

[4] R. Rosen, Life Itself (New York, Columbia University Press, 1991).

[5] A.M. Turing, Philosophical Transactions of the Royal Society B 237, 37 (1952).

[6] S.A. Kauffman, The Origin of Order: Self-Organization and Selection in Evolution (New York, Oxford University Press, 1993).

[7] A.C. Algarte, A.R. Vasconcellos e R. Luzzi, Physica Status Solidi (b) 173, 487 (1992).

[8] A.F. Fonseca, M.V. Mesquita, A.R. Vasconcellos e R. Luzzi, Journal of Chemical Physics 112, 3967 (2000).

[9] R.A. Fisher, Philosophical Transactions of the Royal Society London A 222, 309 (1922).

[10] R. Balian, American Journal of Physics 67, 1078 (1999).

[11] P.T. Landsberg, Brazilian Journal of Physics 29, 46 (1999).

[12] G.M. Zaslavsky, Physics Reports 371, 461 (2002).

[13] M.F. Shlesinger, G.M. Zaslavsky e U. Frish, Levy Flights and Related Topics in Physics (Berlin, Springer, 1985). 
[14] W. Ebeling, Statistical Physics and Thermodynamics of Nonlinear Nonequilibrium Systems (Singapore, World Scientific, 1993).

[15] R. Feistel e W. Ebeling, Evolution of Complex Systems (Dordrecht, Kluwer Academic, 1989).

[16] W. Ebeling, Physica A 182, 108 (1992).

[17] C. Beck e E.G.D. Cohen, arXiv:cond-matl0205097 (2002).

[18] J. Havrda e F. Charvat, Kybernetica 3, 30 (1967).

[19] S. Abe e Y. Okamoto, Nonextensive Statistical Mechanics and its Application (Berlin, Springer, 2001).

[20] C. Tsallis, Journal of Statistical Physics 52, 479 (1988).

[21] A. Renyi, em Proceedings of the Fourth Berkeley Symposium on Mathematical Statistics and Probability (University of California, Berkeley, 1961).

[22] A. Renyi, Probability Theory (Dover Publications, North Holland, 1970).

[23] F. Takensand e E. Verbitski, Nonlinearity 11771 (1998).

[24] P. Grassberger e I. Procaccia, Physical Review A 28, 2591 (1983).

[25] P. Grassberger, Physica D 14, 365 (1985).

[26] P. Jizba e T. Arimitzu, Annals of Physics 312, 17 (2004)

[27] B.D. Sharma e D.P. Mittal, Journal of Mathematical Sciences 10, 28 (1975).

[28] V.M. Vasyliunas, Journal of Geophysical Research 73, 2839 (1968).

[29] G. Kaniadakis, Physical Review E 66, 56125 (2002).

[30] R. Luzzi, A.R. Vasconcellos e J.G. Ramos, Predictive Statistical Mechanics: A Non-equilibrium Ensemble Formalism (Kluwer Academic, Dordrecht, 2002).

[31] E.T. Jaynes, in: E. T. Jaynes Papers on Probability, Statistics, and Statistical Physics, editado por R.D. Rosenkrantz (Reidel-Kluwer Academic, Dordrecht, 1983).

[32] E.T. Jaynes, in: Frontiers of Non-Equilibrium Statistical Physics, editado por G.T. Moore e M.O. Scully (Plenum, New York, 1986), p. 33

[33] C.E. Shannon e W. Weaver, The Mathematical Theory of Communication (University Illinois Press, Urbana, 1948).

[34] R.T. Cox, The Algebra of Probable Inference (John Hopkings Press, Baltimore, 1961).

[35] E.T. Jaynes, Probability Theory: The Logic of Science (Cambridge University Press, Cambridge, 2002).

[36] R. Luzzi, A.R. Vasconcellos e J.G. Ramos, Science 298, 1171 (2002).

[37] S. Kullback e R.A. Leibler, The Annals of Mathematical Statistics 22, 79 (1951).

[38] R. Balian e N.L. Balazs, Annals of Physics 179, 97 (1987).

[39] J.W.T. Grandy, Principles of Statistical Mechanics: Equilibrium Theory (Reidel-Kluwer Academic, Dordrecht, 1987).

[40] J.W.T. Grandy, Principles of Statistical Mechanics: Nonequilibrium Phenomena (Reidel-Kluwer Academic, Dordrecht, 1988).

[41] P.S. Laplace, Essay Philosophique sur les Probabilities (Bachelier, Paris, 1825)

[42] E.T. Jaynes, in: The Maximum Entropy Formalism, editado por M. Tribus e R.D. Levine (MIT Press, Cambridge, 1978), p. 15.

[43] L. Onsager, Physical Review 37, 405 (1931).
[44] H.G.B. Casimir, Review of Modern Physics 17, 343 (1945).

[45] E.T. Jaynes, Physical Review 106, 620 (1957).

[46] E.T. Jaynes, Physical Review 108, 171 (1957).

[47] E.T. Jaynes, in: Maximum Entropy and Bayesian Methods, editado por J. Skilling, (Kluwer Academic, Dordrecht, 1989), p. 1.

[48] E.T. Jaynes, in: Maximum Entropy and Bayesian Methods, editado por W.T. Grandy e L.H. Schick,(Kluwer Academic, Dordrecht, 1991) p. 1.

[49] E.T. Jaynes, in: Physics and Probability, editado por W.T. Grandy e P.W. Milonni, (Cambridge University Press, Cambridge, 1993), p. 261.

[50] H. Jeffreys, Probability Theory (Clarendon, Cambridge, 1961).

[51] H. Jeffreys, Scientific Inference (Cambridge University Press, Cambridge, 1973).

[52] R. Luzzi, A.R. Vasconcellos e J.G. Ramos, International Journal of Modern Physics B 14, 3189 (2000).

[53] R. Luzzi, A.R. Vasconcellos e J.G. Ramos, Fortschritte der Physik/Progress of Physics 38, 887 (1990).

[54] J. Uffink, Studies in History and Philosophy of Modern Physics 26, 223 (1995).

[55] W. Elsasser, Physical Review 52, 987 (1937).

[56] R. Landauer, Physical Review A 12, 636 (1975).

[57] M. Bunge, in: The Flight from Science and Reason, editador por P.R. Gross, N. Levit e M. Lewis (New York Academy of Science, New York, 1996), v. 665.

[58] J. Bricmont, in: The Flight from Science and Reason, editado por P.R. Gross, N. Levit e M. Lewis (New York Academy of Science, New York, 1996).

[59] P.W. Anderson, Physics Today 45, 9 (1992).

[60] W. Heisenberg, The Physical Conception of Nature (Hutchinson, London, 1958).

[61] W. Heisenberg, in: Across the Frontiers, editado por R.N. Anshen e R. Harper, (Ox Bow Pr, New York, 1975), p. 184.

[62] M. Born, Experiment and Theory in Physics (Dover, New York, 1956).

[63] S.W. Hawking, 1990 - Yearbook of Science and Future (Encyclopaedia Britannica, Chicago, 1989).

[64] R. Luzzi, A.R. Vasconcellos e J.G. Ramos, La Rivista del Nuovo Cimento 29, 1 (2006).

[65] L. Sklar, Physics and Chance: Philosophical Issues in the Foundations of Statistical Mechanics (Cambridge University Press, Cambridge, 1993).

[66] B.C.V. Fraassen, British Journal for the Philosophy of Science 32, 75 (1981).

[67] B.C.V. Fraassen, R.I.G. Hughes e G. Harman, British Journal for the Philosophy of Science 37, 453 (1986).

[68] B.C.V. Fraassen, Laws and Symmetry (Claredon, Oxford, 1989).

[69] J.N. Kapur e H.K. Kesavan, Entropy Optimization Principles with Applications (Boston Academic, Boston, 1992).

[70] D.N. Zubarev, V.N. Morozov e G. Röpke, Statistical Mechanics of Nonequilibrium Processes: Basic Concepts, Kinetic Theory (Akademie Verlag Wiley-VHC, Berlin, 1996).

[71] R. Luzzi, A.R. Vasconcellos, J.G. Ramos e C.G. Rodrigues, Theoretical and Mathematical Physics 194, 4 (2018). 
[72] A.L. Kuzemsky, Teoreticheskaia i Matematichaskaia Fizika 194, 71 (2018).

[73] V.G. Morozov, Teoreticheskaia i Matematichaskaia Fizika 194, 127 (2018).

[74] A. Hobson, The Journal of Chemical Physics 45, 1352 (1966).

[75] A. Hobson, American Journal of Physics 34, 411 (1966).

[76] L.S. Garcia-Colin, A.R. Vasconcellos e R. Luzzi, Journal of Non-Equilibrium Thermodynamics 19, 24 (1994).

[77] R. Luzzi, A.R. Vasconcellos e J.G. Ramos, Statistical Foundations of Irreversible Thermadynamics (TeubnerBertelsmann Springer, Stuttgart, 2000).

[78] B. Robertson, Physical Review 144, 151 (1966).

[79] B. Robertson, Physical Review 160, 175 (1967).

[80] L. Lauck, A.R. Vasconcellos e R. Luzzi, Physica A 168, 789 (1990).

[81] C.G. Rodrigues, A.R. Vasconcellos e R. Luzzi, Transport Theory and Statistical Physics 29, 733 (2000).

[82] R. Luzzi e A.R. Vasconcellos, Journal of Statistical Physics 23, 539 (1980).

[83] A.R. Vasconcellos, M.V. Mesquita e R. Luzzi, Physical Review Letters 80, 2008 (1998).

[84] C.G. Rodrigues, A.R. Vasconcellos e R. Luzzi, Solid State Communications 140, 435 (2006).

[85] C.G. Rodrigues, F.S. Vannucchi e R. Luzzi, Advanced Quantum Technologies 1, 201800023 (2018).

[86] J.G. Ramos, A.R. Vasconcellos e R. Luzzi, Fortschritte der Physik/Progress of Physics 43, 265 (1995).

[87] J.R. Madureira, A.R. Vasconcellos, R. Luzzi, J. CasasVazquez e D. Jou, Journal of Chemical Physics 108, 7568 (1998).

[88] T. Dedeurwaerdene, J. Casas-Vazquez, D. Jou e G. Lebon, Physical Review E, 53, 498 (1996).

[89] D. Jou, J. Casas-Vazquez, J.R. Madureira, A.R. Vasconcellos e R. Luzzi, Journal of Chemical Physics 116, 1571 (2001).

[90] C.A.B. Silva, C.G. Rodrigues, J.G. Ramos e R. Luzzi, Physical Review E 91, 063011 (2015).

[91] A.R. Vasconcellos, A.R.B. Castro, C.A.B. Silva e R. Luzzi, AIP Advances 3, 072106 (2013).

[92] C.G. Rodrigues, A.R. Vasconcellos e R. Luzzi, The European Physical Journal B 86, 200 (2013).

[93] A.R. Vasconcellos, A.A.P. Silva, R. Luzzi, Casas-Vázquez J e D. Jou, Physical Review E 88, 042110 (2013).

[94] E.T. Jaynes, American Journal of Physics 33, 391 (1965). 